\title{
Nature of Complex Network of Dengue Epidemic as a Scale-Free Network
}

\author{
Hafiz Abid Mahmood Malik ${ }^{1}$, Faiza Abid², Nadeem Mahmood ${ }^{3}$, Mohamed Ridza Wahiddin ${ }^{4}$, Asif Malik \\ 'Department of Computer Science, AMA International University, Salmabad, Bahrain \\ ${ }^{2}$ Department of Computer Science, King Khalid University, Abha, Kingdom of Saudi Arabia \\ ${ }^{3}$ Department of Computer Science, Faculty of Computer Science, University of Karachi, Sindh, Pakistan \\ ${ }^{4}$ Faculty of Information and Communication Technology, International Islamic University, Selangor, Malaysia \\ ${ }^{5}$ Department of Sciences, Government College University, Lahore, Pakistan
}

Objectives: Dengue epidemic is a dynamic and complex phenomenon that has gained considerable attention due to its injurious effects. The focus of this study is to statically analyze the nature of the dengue epidemic network in terms of whether it follows the features of a scale-free network or a random network. Methods: A multifarious network of Aedes aegypti is addressed keeping the viewpoint of a complex system and modelled as a network. The dengue network has been transformed into a one-mode network from a two-mode network by utilizing projection methods. Furthermore, three network features have been analyzed, the power-law, clustering coefficient, and network visualization. In addition, five methods have been applied to calculate the global clustering coefficient. Results: It has been observed that dengue epidemic follows a powerlaw, with the value of its exponent $\gamma=-2.1$. The value of the clustering coefficient is high for dengue cases, as weight of links. The minimum method showed the highest value among the methods used to calculate the coefficient. Network visualization showed the main areas. Moreover, the dengue situation did not remain the same throughout the observed period. Conclusions: The results showed that the network topology exhibits the features of a scale-free network instead of a random network. Focal hubs are highlighted and the critical period is found. Outcomes are important for the researchers, health officials, and policy makers who deal with arbovirus epidemic diseases. Zika virus and Chikungunya virus can also be modelled and analyzed in this manner.

Keywords: Dengue Virus, Arboviruse, Epidemics, Big Data, Network Meta-Analysis

Submitted: January 16, 2019

Revised: 1st, April 13, 2019; 2nd, May 16, 2019; 3rd, June 23, 2019

Accepted: July 17, 2019

\section{Corresponding Author}

Hafiz Abid Mahmood Malik

Department of Computer Science, AMA International University, Salmabad, Bahrain. Tel: +973-17787978,E-mail: hamalik@amaiu. edu.bh (https://orcid.org/0000-0002-1866-1494)

This is an Open Access article distributed under the terms of the Creative Commons Attribution Non-Commercial License (http://creativecommons.org/licenses/by$\mathrm{nc} / 4.0 /$ ) which permits unrestricted non-commercial use, distribution, and reproduction in any medium, provided the original work is properly cited.

(c) 2019 The Korean Society of Medical Informatics

\section{Introduction}

Various kinds of real world complex systems including physical, biological, and social systems can be represented in terms of complex networks. Some important examples are the World Wide Web, electric power grids, scientific collaboration networks, airport networks, the Hajj network, and social networks of friendship [1-4]. In the fields of medicine and biology, the complex networks of diseases such as HIV/ AIDS, smallpox, and dengue virus have also been investigated to analyze the spreading phenomenon $[5,6]$. The dengue virus network includes the mosquito 'Aedes aegypti'. Dengue is an arbovirus that spreads infections through mosquitoes 
to humans and infected humans to mosquitoes (other than Aedes species), constituting a complex network. The network of arbovirus epidemic has become a complex phenomenon. In [7], the robustness of the dengue complex network under targeted versus random attack was observed and it was found that targeted attack gives better outcomes in scale-free networks compared to random networks.

While modeling and analyzing the different complex networks, researchers have observed numerous structural characteristics in different real-world organisms [8]. Specifically, in the last few years the research trend has increasingly moved toward analyzing complex systems by creating networks in the form of nodes and links. This facilitates comprehension of both structural and dynamical features of tangible world complex networks. The research and study of these linkages has played a significant role for immunization in epidemics and network tolerance attacks [9]. The authors observed some scale-free features in the dengue epidemic in Singapore [10]. In addition, they analyzed the dengue spreading situation from the perspective of complex networks and modelled the dataset of dengue affected cases in Selangor as a two-mode network, and then projected it to a one-mode network $[5,11]$. A dataset of dengue patients has been obtained from the Ministry of Health (MoH) Malaysia, and is provided in Appendix 1. The data are anonymous to protect patients' privacy. The network projection is performed by three methods (weighted Newman, sum, and binary) and the power-law exponent $(\gamma)$ is calculated, which is an important step to see the deportment of a scale-free network [11].

The existence of a power-law posture is perceived in numerous varieties of complex networks, including metabolic networks, systems of lung inflation, sun motion, and the light from galaxies and the water flow through river [12]. Thus, the power-law has been utilized for discovering complex environments by many researchers in various research areas and contexts.

The power-law is quite different from a bell-shaped normal distribution. A scale-free graph [8] follows a power-law form as given below:

$$
\mathrm{P}(k) \sim k^{-\gamma}
$$

where $k$ represents the degree of a node, the probability of node degree distribution is represented by $\mathrm{P}(k)$, and $\gamma$ (gam$\mathrm{ma}$ ) is a scaling exponent, which is a statistical parameter that is called a connectivity distribution exponent. In reality, $\gamma$ does not depend on a specific scale of network that is why; it is called a scale-free parameter. Also, the value of $\gamma$ has been confirmed in many research studies to approximately range from 2 to 3 . R-project has been utilized to find $\gamma$ and other graphical visualizations in this research.

Scale-free network comprises two main characteristics based on the Barabási-Albert model: growth and preferential attachment. The remainder of this paper is structured as follows: Section I-1 provides the background of the study. In Sections II and III, the methodology is presented and the results are discussed. Finally, Section IV gives conclusions of this research and directions for future work.

\section{Background}

Dengue fever is a major disease in tropical regions of the world. Approximately 25 million people are in danger due to mosquito borne dengue fever $[13,14]$. It is caused by an arbovirus, Aedes aegypti, which is the primary vector of dengue virus [14]. DENV-1, DENV-2, DENV-3, and DENV-4 are four serotypes that have been found in this disease [14$18]$.

\section{1) Dengue in Malaysia}

MoH Malaysia published a report in 2015 noting 107,079 dengue cases with 293 deaths while there were 43,000 dengue cases with 92 deaths in 2013 [19-21]. According to the $\mathrm{MoH}$ Malaysia, the presence of dengue has been growing rapidly since 2012 [22,23]. The rapid spread of dengue virus has become more harmful and tackling this matter should be considered urgent. Dengue epidemic is an important field of research and many researchers are investigating this phenomenon from different viewpoints [18,24,25]. In light of the importance of this issue and with the aim of working toward a remedy, the authors have modelled the dengue problem in Malaysia by utilizing the two-mode network technique [11].

\section{2) Modeling of dengue epidemic network}

Researchers have been utilizing the two-mode network technique in many fields, such as collaborative work and movieactor networks [26-28]. The authors of this paper have utilized the two-mode network technique in the dengue mosquito network [11]. In this network primary nodes are represented by L1, L2, L3, and L4 and secondary set of nodes are represented by $\mathrm{W} 1, \mathrm{~W} 2, \mathrm{~W} 3$, and W4. A two-mode network is usually converted into a one-mode network by projection for better analysis [28]. The current work is an enhanced form and continuation of our previous work [11]. Analysis of the network is based on the power-law behavior, 
clustering coefficient, and network visualization, and most importantly we observed the characteristics of the Aedes aegypti network from the viewpoint of a scale-free network and random network. In general, there are various types of networks and different ways to destroy those networks. It is very important that before attacking any network, the topology of the network should be understood. Similarly, to break down the dengue network, it must be clarified whether it should be treated as a scale-free network or a random network

\section{Methods}

\section{Network Analysis}

This section presents an analysis of the weighted two-mode dengue network, which has been performed from the perspective of dengue cases that appeared in different locations in Selangor. After utilizing the projection ways (binary, sum, and weighted Newman) and on the basis of their outcomes, the weighted Newman method has been observed to provide the best fit for this dataset. By this method, the dataset lost the minimum weighted information [11]. From the given dataset, the power-law exponent $(\gamma)$ is calculated and the results showed the trend towards a power-law. It should be noted that a scale-free network follows a power-law [8]. In this research, the results show the characteristics of a scalefree network and particularly the topology of this complex dengue network is in a power-law form.

To see the graphical trends of the power-law, graphs are formed utilizing the dataset (bipartite) of Gombak, Hulu Langat, and Petaling, where numbers of dengue cases are shown in effected localities, respectively. Furthermore, the clustering coefficient is calculated to observe the deviation of the network.

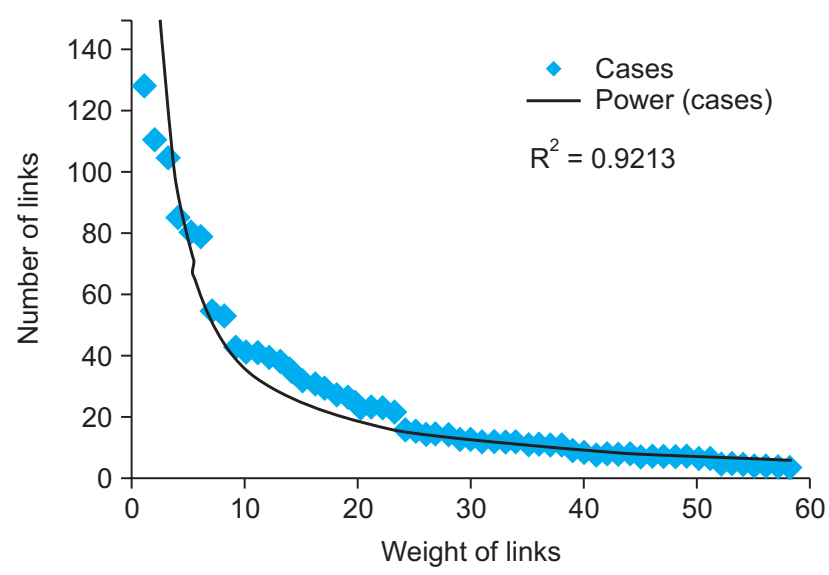

Figure 1. Dengue cases in Gombak, Selangor (2013-2014).

\section{Results}

\section{Power-Law Behavior}

In Figure 1, the $\mathrm{x}$-axis shows the weight of links and the $\mathrm{y}$ axis shows the number of links. Dengue cases in different localities of Gombak that occurred during the observed years are represented on a linear scale. The figure displays powerlaw behavior. It clearly indicates that the human populations in a few localities were highly affected by the dengue virus in Selangor, Malaysia, during the given period.

There are 58 localities in Gombak that recorded 1487 dengue cases in total in the given period. The highest number of dengue cases $(=128)$ was registered in location no. 18 whereas only four cases were observed in location no. 2. In Figure 2, Hulu Langat, 185 localities were affected compared to Petaling, where 243 dengue affected localities were registered. It is interesting that in these three areas only a few localities were attacked repeatedly by Aedes aegypti, whereas in other localities this mosquito attacked once or twice in a year. This showed the dengue has effected within this particular area. Similarly, there are a few areas that were highly affected by the dengue virus. In very few places the dengue appearance is high whereas the majority of localities have a small number of dengue cases. There is consequently a need to place greater focus on these few areas to control this disease. On the other side, identification of focal nodes in a complex networks is an important issue for researchers and scientists. Specifically, if the central node is identified, it will potentially make it possible to control the flow of other nodes. Moreover, via that node, other nodes can be captured very quickly. Hence, targeting the areas in which dengue virus is appearing repeatedly and affecting large populations may be a more helpful means to find and control the central node.

In Figures $1-3, \mathrm{R}^{2}$ is the coefficient of determination. This

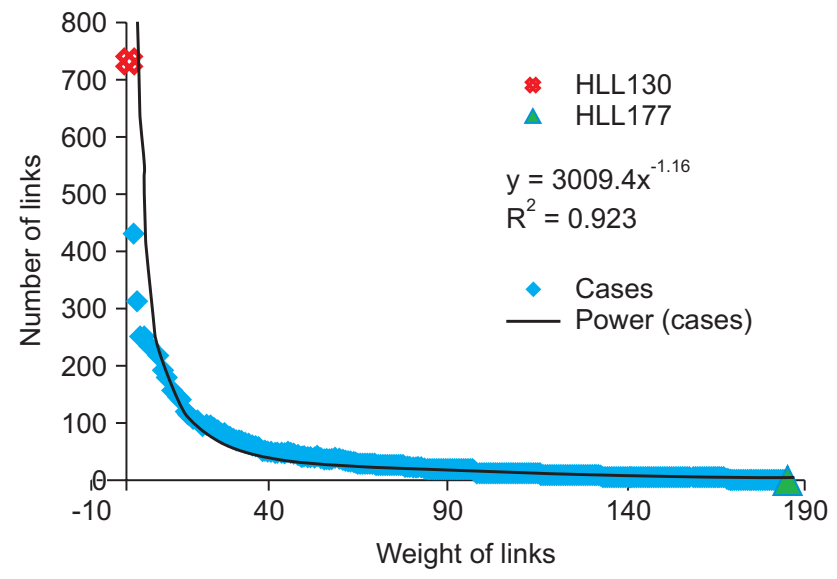

Figure 2. Dengue cases in Hulu Langat, Selangor (2013-2014). 
statistical measure shows how good the regression line estimates the real data points. $\mathrm{R}^{2}$ provides information on the goodness of fit of a model. Here, $\mathrm{R}^{2}=0.9213,0.923$, and 0.9102 specify that the regression line perfectly fits the data, respectively.

The coefficient of determination is calculated as:

$$
\mathrm{R}^{2}=1-\frac{S S_{\text {res }}}{S S_{\text {tot }}}
$$

where $S S_{t o t}$ represents the total sum of squares and $S S_{\text {res }}$ is the residual sum of squares.

In Figure 2, the $\mathrm{x}$-axis shows the weight of links and the $\mathrm{y}$ axis shows the number of links. The number of dengue cases in different localities of Hulu Langat that were included in this network during the given period. The weight of links as dengue cases in different localities is shown on a linear scale. Out of 185 dengue affected localities, 7,854 dengue cases were registered. The highest number of cases $(=729)$ was recorded in the location HL130, whereas the smallest number of cases $(=4)$ was recorded in location HL177.

In Figure 3, the $\mathrm{x}$-axis shows the weight of links and the $y$-axis shows the number of links. The numbers of dengue cases in different localities of Petaling are displayed on a linear scale during the period 2013-2014. It can be grasped that there are few localities that registered an extraordinary number of cases. Out of 243 affected localities of Petaling, 15,261 dengue cases were recorded throughout the given period. The highest number of dengue cases $(=3,107)$ was registered in Petaling (location no. 126). The smallest number, four cases, was observed in Petaling (location no. 240 and 243).

In the literature, some examples of scale-free network are presented, where the power-law exponent has different values. Researchers considered these networks to be scalefree $[1,8]$. Investigations of the topology of complex systems

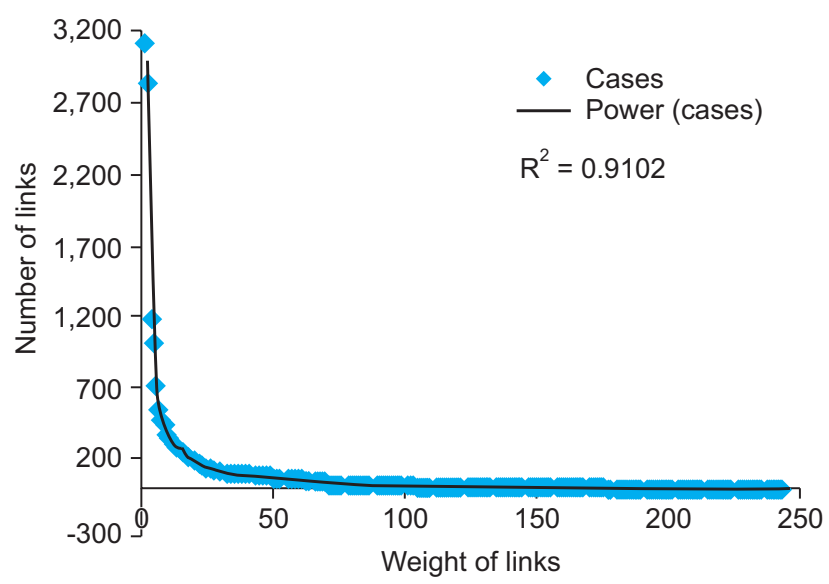

Figure 3. Dengue cases in Petaling, Selangor (2013-2014). from different domains of life have shown interesting results. For example, Barabasi and Bonabeau [8] modelled World Wide Web pages and their hyper-links and brought the idea of scale-free network with power-law exponents $\gamma_{i n}=2.1$ and $\gamma_{\text {out }}=2.7$, where $\gamma_{\text {in }}$ and $\gamma_{\text {out }}$ are in-degree and out-degree, respectively. In 2001, Liljeros et al. [29] modelled and investigated human sexual connections as a network. Researchers found this societal occurrence to be scale-free and showed that it follows the power-law form (where $\gamma_{f}=2.54$ for females and $\gamma_{m}=2.31$ for males). Newman formed a scientific association network as a two-mode network, where he modelled nodes as scientists and their collaborated papers. Two scientists are linked if they worked on a joint article as primary nodes. He observed the degree distribution of this network in the case of a high energy physics databank, which follows a power-law with the exponent $\gamma=1.2$ [1].

Figure 4 shows the probability distribution of node strength (number of dengue cases in different areas of Selangor) on a log scale, for cases recorded in a given period. R-project has been utilized to calculate the power-law exponent $(\gamma)$. The broken line is the slope of the declining curve and it represents that this network is geographically structured as a scale-free network. $\gamma$ is close to the lower bound of its limit. Here, $\gamma=-2.1$, which indicates a decreasing slope of the distribution. This probability distribution indicates that the distribution follows a power-law; it has geographically organized itself during the given period. The power-law is an important indicator of scale-free network. Moreover, important links are few in number and should be focused on as they have huge weight compared to other links. A scale-free network is very important in solving the epidemic issue. Epidemic diseases can be better controlled by this type of net-

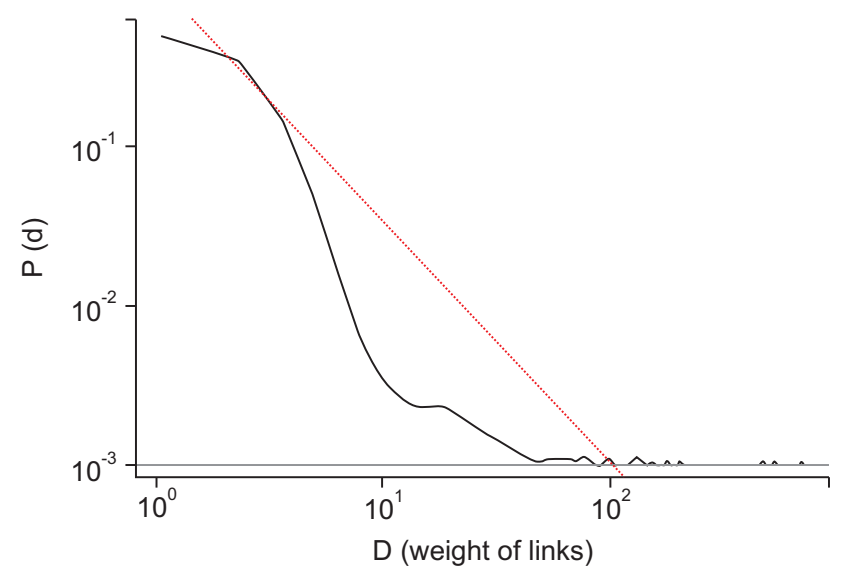

Figure 4. Probability distribution of node strength distribution on log scale in Selangor (2013-2014). The $x$-axis shows the strength of links and the $y$-axis shows the probability distribution of links strength. 
work topology by focusing on main hubs (nodes), compared to a random distribution.

Link's density is shown in Figure 5, where the $\mathrm{x}$-axis represents the number of links and the y-axis shows the weight of links on a linear scale. It specifies that some links in this two-mode network highly affect the whole network. The majority of nodes have weight below 200, whereas a minority of nodes has high weight in terms of dengue cases that appeared in these nodes.

In Figure 6, a weekly comparison is shown among 6 dengue affected districts in Selangor. This graph indicates the most crucial time period when the attack of Aedes aegypti was at its peak. It can be observed from the graph that Petaling is the most affected area followed by Hulu Langat. The human populations of Gombak, Sepang, Hulu Selangor, and Klang have also been victims of Aedes aegypti. The dataset showed the peak activity was from December 2013 till the end of February 2014. These twelve weeks were the most critical in these two highly affected districts. These districts had high infection rates in these 9 weeks compared to the other 3 weeks

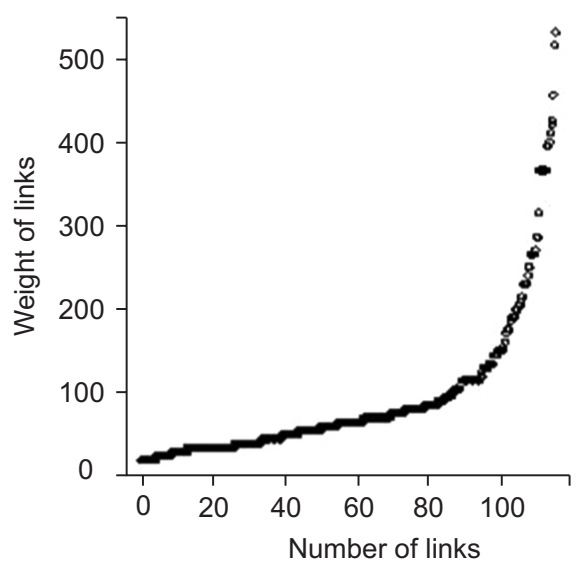

Figure 5. Links density using weighted Newman method of projection. in the year. For the remaining four districts, the time series suggests activity without a clear, sustained epidemic burst between October 20, 2013 and October 18, 2014. Sepang, for instance, appears to have higher activity from October 2013 to the end of December 2013, without any significant activity in 2014. Gombak represented an isolated peak in the 25th week of 2014 and Hulu Selangor showed slightly elevated activity by the end of 2014 (41st week onwards). It is observed that out of 12 months, these 3 months showed the highest rate of dengue infections. It can be concluded that, apart from the importance of focal nodes, time duration is also important, as 3 months showed the highest rate, and also showed power-law resemblance. This feature also indicates a scale-free network.

\section{Clustering Coefficient}

The global clustering coefficient has been generalized to weighted two-mode networks with weighted links [28]. The value can be constructed on the weights of links, and can be defined utilizing the four methods as a one-mode weighted clustering coefficient [27]. For the weighted two-mode network, global clustering coefficient is defined as follows:

$$
\mathrm{C}^{\star \mathrm{w}}=\frac{\text { (Total value of closed 4-paths) }}{\text { (Total values of 4-paths) }}=\frac{\sum \tau^{\star} \Delta \mathrm{w}}{\sum \tau^{\star} \mathrm{W}}
$$

where $\tau^{*} w$ represents the values of 4 -paths and $\tau^{*} \Delta \mathrm{w}$ shows the value of these 4-paths that are closed by being part of at least one 6-cycle (i.e., a loop of six links with five nodes).

Here, the global clustering coefficient is computed for the weighted two-mode network using five methods, i.e. binary $(\mathrm{Bi})$, arithmetic mean (AM), geometric mean (GM), maximum (Max) and minimum (Min).

In Figure 7, the global clustering coefficient is shown in two-mode networks by utilizing the above-mentioned five methods. The results showed that the network of localities is clustered, where dengue cases are considered as the weight of

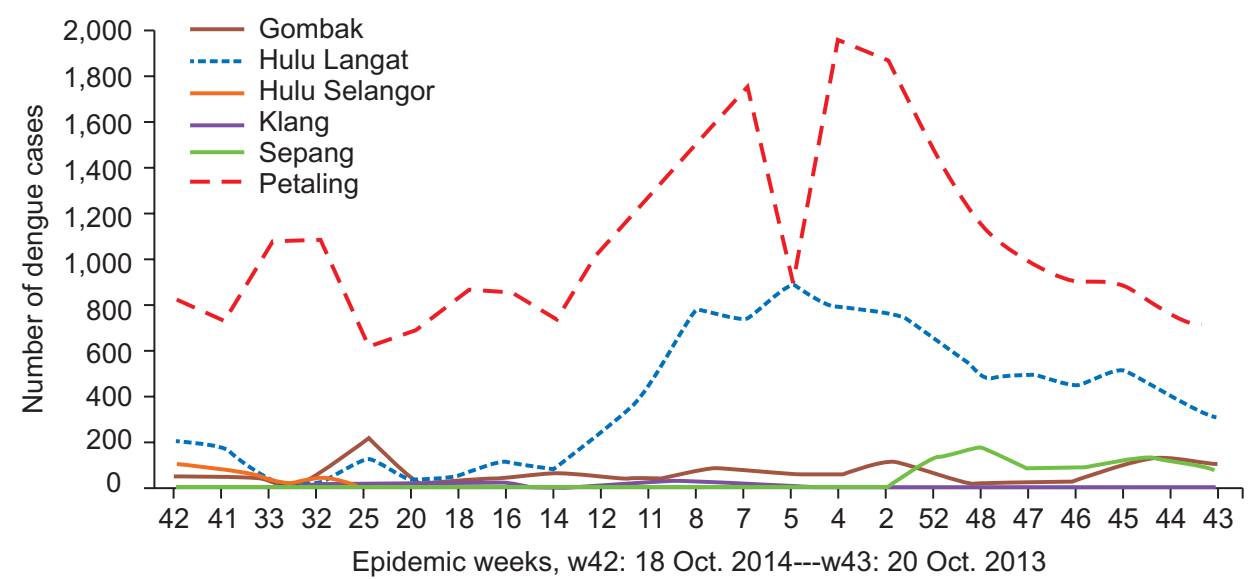

Figure 6. Week-wise comparison among six districts (October 20, 2013 to October 18 , 2014). 


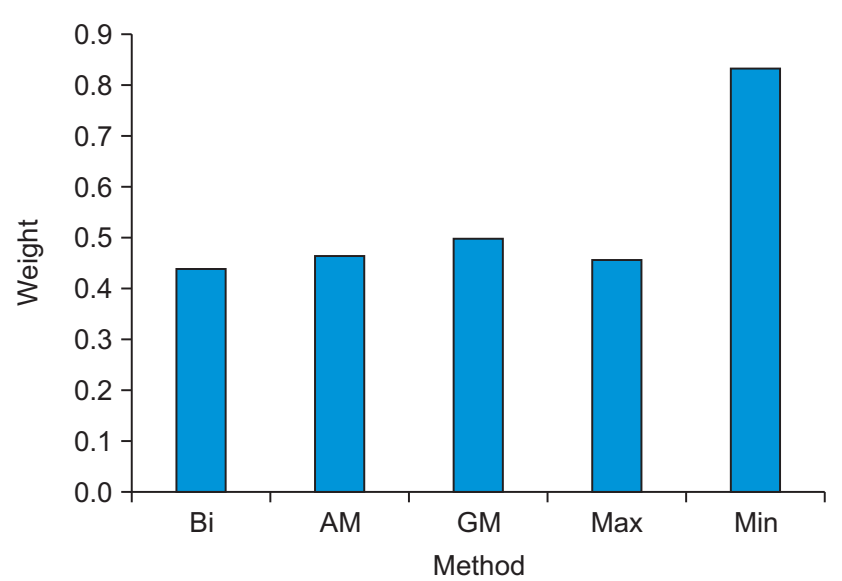

Figure 7. Different methods of global clustering coefficients (comparison) in two-mode network. Bi: binary, AM: arithmetic mean, GM: geometric mean, Max: maximum, Min: minimum.

the links. It can be observed that a few links have very high weight and, because of this, the minimum method showed the highest value $(=0.85)$, which also showed resemblance to a scale-free network. The results of the binary method do not include the weight of links to complete 4-paths. On the other hand, the maximum method showed a lower value compared to all other weighted methods (excluding binary) because the majority of links have smaller weight. There are few links having high weight in the network, and for this reason the minimum method represented high values of the clustering coefficient compared to the other methods. In addition, AM displayed an average value compared to other methods. GM meanwhile produced higher values than Max and AM because this method also closes the 4-paths based on GM. Therefore, in terms of the global clustering coefficient, the minimum method is the most appropriate for use in this network. The results obtained by this method indicate that the weighted distribution is very inhomogeneous.

\section{Network Visualization from Localities Perspective}

Figure 8 presents a graphical view of the dengue network that is plotted in igraph package of R-project. The node ID numbers $1,5,15,30,36,38,39$, and 40 are very significant and should be focused on for treatment. This is due to their degree and their role as bridges between different clusters of nodes. This graphic visualization showed that localities are not properly associated in Gombak with each other by the co-occurrence of weeks [11], also gave the output that not all localities were affected in the observed time frame. Further, few nodes (localities) were working as main hubs. An actual map of Gombak is shown in Figure 9. This provides a geographical representation of dengue affected nodes (localities)

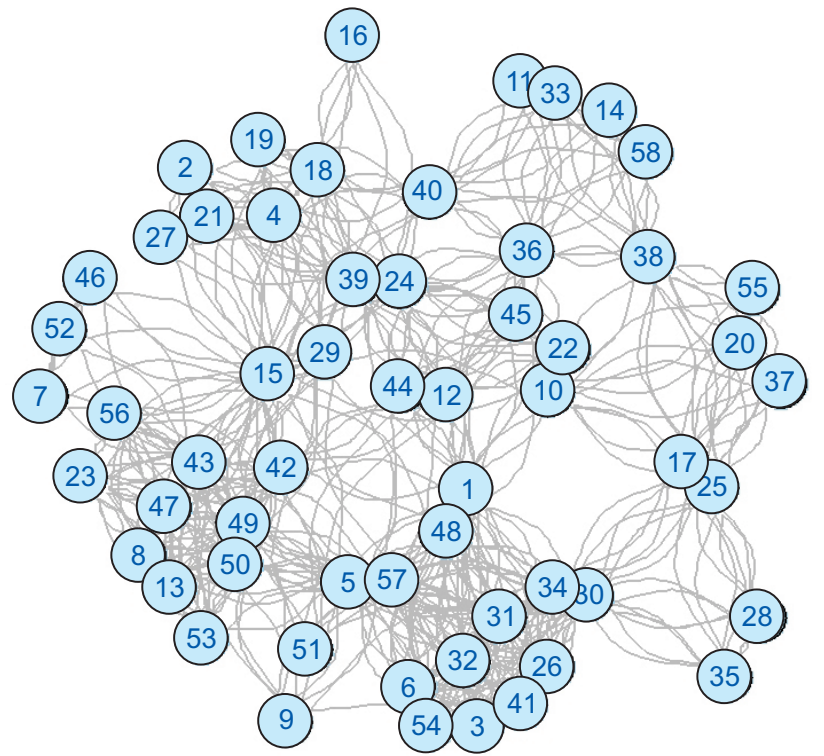

Figure 8. Graphical view of dengue network in Gombak.

in Gombak, Malaysia [11]. It has been observed that there were different clusters, such as Batu Caves, where people suffered greatly due to dengue disease. Grey color nodes represent the critical focal nodes that should be treated first. These nodes also lead this network to be scale-free.

\section{Discussion}

Complex networks have become a rich field of study. Many real-world phenomena are modelled and analyzed as complex networks. The locale of this study is Selangor, a state of Malaysia. Here, the dengue epidemic issue has been modelled by considering a two-mode network and the given dataset of dengue affected cases was investigated. A power-law exponent $(\gamma)$ has been calculated and discussed on the basis of output obtained from the projection methods. The results of network metrics, clustering coefficient, and gamma exhibit the topology of the dengue epidemic as a scale-free network. Furthermore, the dengue situation did not remain the same throughout the year, and it was found that a 12 -week period was more crucial and showed a power-law form. The global clustering coefficient of localities network revealed that this network is clustered in terms of dengue cases as the weight of the links. The findings of this study showed the overall trend of this network as a scale-free instead of random network. These outcomes can help health official policy makers to deal with the dengue virus by keeping in view its scale-free nature. The outcomes highlight focal hubs that can be inspected in terms of cleanliness, immunization, and how the dengue virus can be avoided or controlled. In the future, 


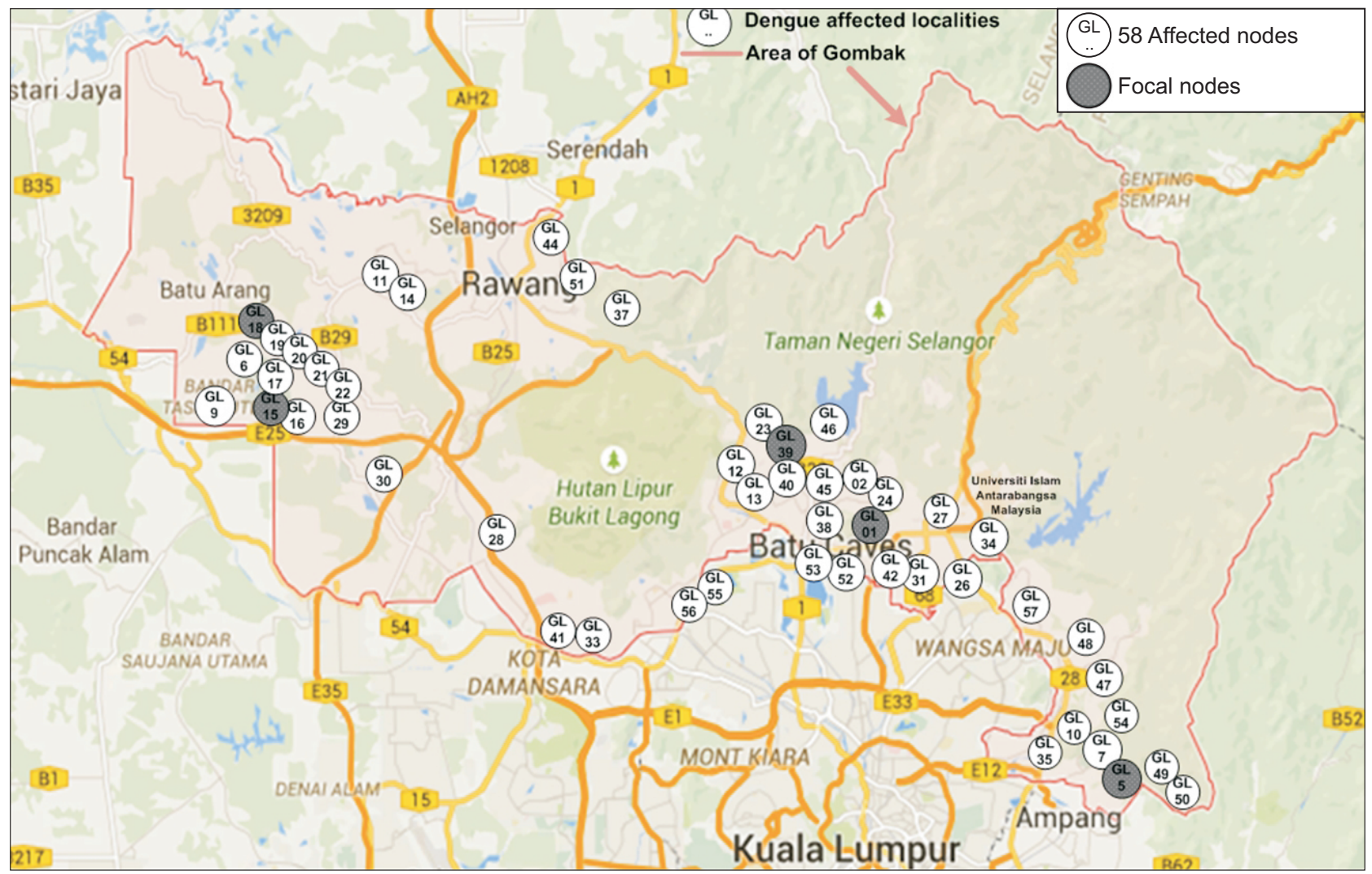

Figure 9. Real map of Gombak, Malaysia. The map showed dengue affected noedes (GL is Gombak, location no. 1 to 58), image captured from Google maps and modified.

the impact of the genetically modified mosquito (GMM) technique can be introduced in focal nodes as an external factor for the treatment of harmful effects of Aedes Aegypti. Furthermore, the GMM technique would be less costly and more effective when applied to a scale-free network compared to a random network. The methods and results of this research are also important for researchers and scientists who deal with arbovirus epidemics, such as the Zika and Chikungunya viruses.

\section{Conflict of Interest}

No potential conflict of interest relevant to this article was reported.

\section{Acknowledgments}

The authors would like to express their gratitude to AMA International University Bahrain, for providing administrative and technical support.

\section{ORCID}

Hafiz Abid Mahmood Malik (http://orcid.org/0000-0002-1866-1494)
Faiza Abid (http://orcid.org/0000-0001-9658-6066)

Nadeem Mahmood (http://orcid.org/0000-0001-6365-7649)

Mohamed Ridza Wahiddin (http://orcid.org/0000-0002-0244-2115)

Asif Malik (http://orcid.org/0000-0003-3545-5024)

\section{References}

1. Newman ME. The structure of scientific collaboration networks. Proc Natl Acad Sci U S A 2001;98(2):404-9.

2. Malik HA, Mahmood N, Usman MH, Rziwan K, Abid F. Analysis of airport network in Pakistan utilizing complex network approach. Int J Adv Comput Sci Appl 2019;10(1):404-10.

3. Malik HA, Mahmood N, Usman MH, Abid F. Unweighted network study of Pakistani airports. Proceedings of 2019 2nd International Conference on Computing, Mathematics and Engineering Technologies (iCoMET); 2019 Jan 30-31; Sukkur, Pakistan. p. 1-6.

4. Malik HA, Abid F, Gilal AR, Raja AS. Use of cloud computing in Hajj crowed management and complex systems. Proceedings of 2017 4th IEEE International Conference on Engineering Technologies and Applied Sciences (ICETAS); 2017 Nov 29-Dec 1; Salmabad, Bahrain. pp. 1-5. 
5. Malik HA, Mahesar AW, Abid F, Wahiddin MR. Twomode complex network modeling of dengue epidemic in Selangor, Malaysia. Proceedings of the 5th International Conference on Information and Communication Technology for the Muslim World (ICT4M); 2014 Nov 17-18; Kuching, Malaysia. p. 1-6.

6. Kim PS, Lee PP, Levy D. Basic principles in modeling adaptive regulation and immunodominance. In: Ledzewicz U, et al., editors. Mathematical methods and models in biomedicine. New York (NY): Springer Science \& Business Media; 2013. p. 33-57.

7. Malik HA, Abid F, Wahiddin MR, Bhatti Z. Robustness of dengue complex network under targeted versus random attack. Complexity 2017;2017;2515928.

8. Barabasi AL, Bonabeau E. Scale-free networks. Sci Am 2003;288(5):60-9.

9. Pastor-Satorras R, Vazquez A, Vespignani A. Dynamical and correlation properties of the internet. Phys Rev Lett 2001;87(25):258701.

10. Massad E, Ma S, Chen M, Struchiner CJ, Stollenwerk N, Aguiar M. Scale-free network of a dengue epidemic. Appl Math Comput 2008;195(2):376-81.

11. Malik HA, Mahesar AW, Abid F, Waqas A, Wahiddin MR. Two-mode network modeling and analysis of dengue epidemic behavior in Gombak, Malaysia. Appl Math Model 2017;43:207-20.

12. Uddin S, Murshed ST, Hossain L. Power-law behavior in complex organizational communication networks during crisis. Physica A Stat Mech Appl 2011;390(15):284553.

13. Soewono E, Supriatna AK. A two-dimensional model for the transmission of dengue fever disease. Bull Malaysian Math Sci Soc 2001:24(1):49-97.

14. Pessanha JE. Risk assessment and risk maps using a simple dengue fever model. Dengue Bull 2012;36:73-86.

15. Ximenes R, Massad E. Modelling the risk of dengue for tourists in Rio de Janeiro during the FIFA confederation cup in Brazil 2013 [Internet]. Sao Paulo, Brazil: University of Sao Paulo; 2013 [cited at 2019 Jul 1]. Available from: https://www.fields.utoronto.ca/programs/scientific/13-14/ BIOMAT/presentations/Ximenes.pdf.

16. Coutinho FA, Burattini MN, Lopez LF, Massad E. Threshold conditions for a non-autonomous epidemic system describing the population dynamics of dengue. Bull Math Biol 2006;68(8):2263-82.

17. Malik H, Waqas A, Abid F, Gilal A, Mahessar A, Koondar Y. Complex network of dengue epidemic and link prediction. Sindh Univ Res J (Sci Ser) 2016;48(4):845-8.

18. Massad E, Ma S, Chen M, Struchiner CJ, Stollenwerk
N, Aguiar M. Scale-free network of a dengue epidemic. Appl Math Comput 2008;195(2):376-81.

19. National Environment Agency. UNITEDengue crossborder data sharing provided countries with timely risk alerts [Internet]. Putrajaya, Malaysia: Ministry of Health; 2014 [cited at 2019 Jul 1]. Available from: http:// www.moh.gov.my/index.php/database_stores/attach_ download/337/573.

20. Ministry of Health. Malaysia health systems research (Volume 1. Contextual analysis of the Malaysia health system) [Internet]. Putrajaya, Malaysia: Ministry of Health; 2016 [cited at 2019 Jul 1]. Available from: http:// www.moh.gov.my/moh/resources/Vol_1_MHSR_Contextual_Analysis_2016.pdf.

21. World Health Organization. Dengue and severe dengue [Internet]. Geneva, Switzerland: World Health Organization; c2019 [cited at 2019 Jul 1]. Available from: https://www.who.int/en/news-room/fact-sheets/detail/ dengue-and-severe-dengue.

22. Ministry of Health Malaysia. Situasi Demam Denggi di Malaysia Bagi Minggu 2/2014 [Internet]. Putrajaya, Malaysia: Ministry of Health; c2019 [cited at 2019 Jul 1]. Available from: http://www.moh.gov.my/index.php/ database_stores/store_view_page/17/465.

23. World Health Organization. Dengue Situation Update Number 441 [Internet]. Manila, Philippines: Regional Office for the Western Pacific Region, World Health Organization; 2014 [cited at 2019 Jul 1]. Available from: https://reliefweb.int/sites/reliefweb.int/files/resources/ dengue.biweekly.15jul2014.pdf.

24. Moheeput K, Goorah SS, Ramchurn SK. Spreading dynamics of a viral infection in a complex network. Int J Med Health Biomed Bioeng Pharm Eng 2013;7(7):3737.

25. Side S, Noorani MS. A SIR model for spread of dengue fever disease (simulation for South Sulawesi, Indonesia and Selangor, Malaysia). World J Model Simul 2013; 9(2):96-105.

26. Davis A, Gardner BB, Gardner MR. Deep south: a social anthropological study of caste and class. Columbia (SC): University of South Carolina Press; 2009.

27. Barzel B, Barabasi AL. Universality in network dynamics. Nat Phys. 2013;9:673-81.

28. Opsahl T. Triadic closure in two-mode networks: Redefining the global and local clustering coefficients. Soc Netw 2013;35(2):159-67.

29. Liljeros F, Edling CR, Amaral LA, Stanley HE, Aberg Y. The web of human sexual contacts. Nature 2001; 411(6840):907-8. 
Appendix 1. Dataset of dengue patients in Selangor (October 20, 2013 to October 18, 2014)

\begin{tabular}{|c|c|c|c|c|c|c|c|c|c|}
\hline Ref. code & Cases & Ref. code & Cases & Ref. code & Cases & Ref. code & Cases & Ref. code & Cases \\
\hline GL18 & 128 & GL27 & 8 & HLL13 & 76 & HLL36 & 28 & HLL168 & 14 \\
\hline GL5 & 111 & GL57 & 7 & HLL63 & 74 & HLL88 & 28 & HLL52 & 13 \\
\hline GL1 & 105 & GL28 & 7 & HLL89 & 73 & HLL18 & 27 & HLL103 & 13 \\
\hline GL15 & 85 & GL23 & 7 & HLL129 & 71 & HLL73 & 27 & HLL148 & 13 \\
\hline GL39 & 80 & GL21 & 7 & HLL82 & 70 & HLL141 & 27 & HLL16 & 12 \\
\hline GL49 & 79 & GL11 & 7 & HLL139 & 70 & HLL43 & 26 & HLL28 & 12 \\
\hline GL34 & 55 & GL19 & 6 & HLL174 & 66 & HLL142 & 25 & HLL116 & 12 \\
\hline GL56 & 53 & GL16 & 6 & HLL34 & 60 & HLL91 & 24 & HLL132 & 12 \\
\hline GL40 & 43 & GL52 & 5 & HLL12 & 59 & HLL97 & 24 & HLL149 & 12 \\
\hline GL43 & 41 & GL37 & 5 & HLL75 & 54 & HLL101 & 23 & HLL167 & 12 \\
\hline GL24 & 41 & GL10 & 5 & HLL77 & 54 & HLL45 & 22 & HLL35 & 11 \\
\hline GL50 & 40 & GL9 & 4 & HLL152 & 53 & HLL134 & 22 & HLL110 & 11 \\
\hline GL13 & 38 & GL53 & 4 & HLL164 & 52 & HLL138 & 21 & HLL156 & 11 \\
\hline GL17 & 35 & GL29 & 4 & HLL166 & 52 & HLL108 & 20 & HLL172 & 11 \\
\hline GL12 & 32 & GL2 & 4 & HLL24 & 51 & HLL115 & 20 & HLL56 & 10 \\
\hline GL25 & 31 & HLL130 & 729 & HLL165 & 51 & HLL21 & 19 & HLL61 & 10 \\
\hline GL14 & 29 & HLL64 & 435 & HLL182 & 51 & HLL31 & 19 & HLL69 & 10 \\
\hline GL38 & 27 & HLL84 & 317 & HLL185 & 50 & HLL68 & 19 & HLL79 & 10 \\
\hline GL47 & 26 & HLL117 & 251 & HLL143 & 48 & HLL74 & 19 & HLL157 & 10 \\
\hline GL8 & 23 & HLL161 & 250 & HLL146 & 46 & HLL114 & 19 & HLL40 & 9 \\
\hline GL51 & 23 & HLL46 & 237 & HLL9 & 45 & HLL136 & 19 & HLL54 & 9 \\
\hline GL36 & 23 & HLL60 & 231 & HLL170 & 45 & HLL17 & 18 & HLL83 & 9 \\
\hline GL42 & 21 & HLL87 & 228 & HLL11 & 44 & HLL29 & 18 & HLL171 & 9 \\
\hline GL54 & 16 & HLL44 & 217 & HLL2 & 43 & HLL41 & 18 & HLL178 & 9 \\
\hline GL26 & 15 & HLL184 & 194 & HLL86 & 43 & HLL55 & 18 & HLL8 & 8 \\
\hline GL6 & 14 & HLL15 & 183 & HLL90 & 42 & HLL153 & 18 & HLL20 & 8 \\
\hline GL4 & 14 & HLL85 & 179 & HLL50 & 41 & HLL98 & 17 & HLL48 & 8 \\
\hline GL22 & 14 & HLL158 & 159 & HLL123 & 41 & HLL1 & 16 & HLL53 & 8 \\
\hline GL48 & 13 & HLL65 & 149 & HLL137 & 40 & HLL113 & 16 & HLL80 & 8 \\
\hline GL41 & 13 & HLL120 & 141 & HLL39 & 39 & HLL121 & 16 & HLL94 & 8 \\
\hline GL45 & 12 & HLL72 & 122 & HLL70 & 39 & HLL133 & 16 & HLL106 & 8 \\
\hline GL44 & 12 & HLL151 & 116 & HLL67 & 38 & HLL147 & 16 & HLL118 & 8 \\
\hline GL30 & 12 & HLL183 & 113 & HLL26 & 36 & HLL154 & 16 & HLL131 & 8 \\
\hline GL3 & 12 & HLL127 & 110 & HLL38 & 36 & HLL181 & 16 & HLL4 & 7 \\
\hline GL7 & 11 & HLL59 & 103 & HLL135 & 35 & HLL105 & 15 & HLL47 & 7 \\
\hline GL58 & 11 & HLL145 & 96 & HLL180 & 34 & HLL144 & 15 & HLL58 & 7 \\
\hline GL20 & 11 & HLL150 & 96 & HLL19 & 31 & HLL7 & 14 & HLL92 & 7 \\
\hline GL35 & 10 & HLL14 & 95 & HLL42 & 31 & HLL30 & 14 & HLL93 & 7 \\
\hline GL32 & 9 & HLL175 & 95 & HLL96 & 31 & HLL51 & 14 & HLL99 & 7 \\
\hline GL31 & 9 & HLL76 & 90 & HLL112 & 31 & HLL57 & 14 & HLL162 & 7 \\
\hline GL55 & 8 & HLL25 & 83 & HLL104 & 30 & HLL78 & 14 & HLL169 & 7 \\
\hline GL46 & 8 & HLL10 & 81 & HLL37 & 29 & HLL126 & 14 & HLL179 & 7 \\
\hline
\end{tabular}


Appendix 1. Continued 1

\begin{tabular}{|c|c|c|c|c|c|c|c|c|c|}
\hline Ref. code & Cases & Ref. code & Cases & Ref. code & Cases & Ref. code & Cases & Ref. code & Cases \\
\hline GL33 & 8 & HLL49 & 79 & HLL163 & 29 & HLL159 & 14 & HLL3 & 6 \\
\hline HLL5 & 6 & PL158 & 237 & PL208 & 65 & PL10 & 27 & PL49 & 13 \\
\hline HLL22 & 6 & PL59 & 227 & PL224 & 65 & PL226 & 27 & PL93 & 13 \\
\hline HLL23 & 6 & PL26 & 223 & PL68 & 63 & PL40 & 26 & PL129 & 13 \\
\hline HLL27 & 6 & PL171 & 206 & PL111 & 63 & PL166 & 26 & PL144 & 13 \\
\hline HLL66 & 6 & PL174 & 192 & PL95 & 61 & PL75 & 24 & PL113 & 12 \\
\hline HLL102 & 6 & PL131 & 181 & PL206 & 59 & PL105 & 24 & PL157 & 12 \\
\hline HLL107 & 6 & PL79 & 170 & PL3 & 55 & PL27 & 23 & PL183 & 12 \\
\hline HLL111 & 6 & PL175 & 166 & PL169 & 55 & PL48 & 23 & PL191 & 12 \\
\hline HLL119 & 6 & PL179 & 153 & PL53 & 49 & PL112 & 23 & PL214 & 12 \\
\hline HLL173 & 6 & PL69 & 150 & PL57 & 47 & PL156 & 23 & PL227 & 12 \\
\hline HLL33 & 5 & PL18 & 145 & PL145 & 46 & PL73 & 22 & PL12 & 11 \\
\hline HLL81 & 5 & PL65 & 136 & PL228 & 45 & PL223 & 22 & PL58 & 11 \\
\hline HLL100 & 5 & PL87 & 136 & PL118 & 44 & PL6 & 21 & PL155 & 11 \\
\hline HLL100 & 5 & PL87 & 136 & PL118 & 44 & PL6 & 21 & PL155 & 11 \\
\hline HLL124 & 5 & PL178 & 135 & PL89 & 43 & PL25 & 21 & PL187 & 11 \\
\hline HLL125 & 5 & PL44 & 130 & PL130 & 43 & PL72 & 21 & PL15 & 10 \\
\hline HLL128 & 5 & PL82 & 90 & PL115 & 20 & PL20 & 8 & PL241 & 5 \\
\hline HLL160 & 5 & PL172 & 89 & PL201 & 20 & PL78 & 8 & PL39 & 4 \\
\hline HLL176 & 5 & PL193 & 85 & PL203 & 20 & PL81 & 8 & PL164 & 103 \\
\hline HLL6 & 4 & PL124 & 83 & PL94 & 19 & PL84 & 8 & PL225 & 102 \\
\hline HLL32 & 4 & PL1 & 82 & PL121 & 19 & PL108 & 8 & PL123 & 100 \\
\hline HLL62 & 4 & PL80 & 82 & PL125 & 19 & PL149 & 8 & PL236 & 100 \\
\hline HLL71 & 4 & PL139 & 80 & PL135 & 19 & PL163 & 8 & PL41 & 98 \\
\hline HLL95 & 4 & PL182 & 79 & PL148 & 19 & PL219 & 8 & PL19 & 95 \\
\hline HLL109 & 4 & PL66 & 73 & PL216 & 19 & PL220 & 8 & PL192 & 30 \\
\hline HLL122 & 4 & PL98 & 72 & PL217 & 19 & PL230 & 8 & PL34 & 29 \\
\hline HLL140 & 4 & PL46 & 70 & PL212 & 18 & PL9 & 7 & PL7 & 28 \\
\hline HLL155 & 4 & PL238 & 70 & PL64 & 17 & PL119 & 7 & PL61 & 28 \\
\hline HLL177 & 4 & PL51 & 65 & PL211 & 17 & PL143 & 7 & PL85 & 21 \\
\hline PL126 & 3107 & PL186 & 43 & PL2 & 16 & PL159 & 7 & PL207 & 21 \\
\hline PL31 & 2826 & PL52 & 42 & PL104 & 16 & PL160 & 7 & PL154 & 9 \\
\hline PL134 & 1174 & PL184 & 42 & PL215 & 16 & PL177 & 7 & PL162 & 9 \\
\hline PL127 & 1022 & PL204 & 42 & PL234 & 16 & PL188 & 7 & PL197 & 9 \\
\hline PL137 & 720 & PL63 & 41 & PL237 & 16 & PL198 & 7 & PL202 & 9 \\
\hline PL54 & 548 & PL106 & 41 & PL43 & 15 & PL213 & 7 & PL209 & 9 \\
\hline PL128 & 488 & PL222 & 40 & PL47 & 15 & PL218 & 7 & PL8 & 8 \\
\hline PL200 & 434 & PL50 & 39 & PL55 & 15 & PL239 & 7 & PL42 & 5 \\
\hline PL114 & 393 & PL185 & 39 & PL221 & 15 & PL242 & 7 & PL150 & 5 \\
\hline PL138 & 345 & PL102 & 37 & PL232 & 15 & PL29 & 6 & PL161 & 5 \\
\hline PL33 & 327 & PL152 & 37 & PL233 & 15 & PL30 & 6 & PL190 & 5 \\
\hline PL17 & 298 & PL100 & 35 & PL122 & 14 & PL35 & 6 & PL195 & 5 \\
\hline
\end{tabular}

Continued on the next page. 
Appendix 1. Continued 2

\begin{tabular}{llllllllcc}
\hline Ref. code & Cases & Ref. code & Cases & Ref. code & Cases & Ref. code & Cases & Ref. code & Cases \\
\hline PL173 & 287 & PL99 & 34 & PL146 & 14 & PL56 & 6 & PL210 \\
PL132 & 282 & PL14 & 33 & PL77 & 10 & PL62 & 6 & - & - \\
PL133 & 275 & PL32 & 33 & PL110 & 10 & PL71 & 6 & - & - \\
PL147 & 114 & PL91 & 33 & PL153 & 10 & PL86 & 6 & - & - \\
PL141 & 113 & PL97 & 33 & PL194 & 10 & PL90 & 6 & - & - \\
PL189 & 113 & PL5 & 32 & PL235 & 10 & PL116 & 6 & - & - \\
PL196 & 110 & PL67 & 32 & PL21 & 9 & PL117 & 6 & - & - \\
PL22 & 109 & PL120 & 32 & PL38 & 9 & PL142 & 6 & - & - \\
PL23 & 109 & PL229 & 32 & PL70 & 9 & PL168 & 6 & - \\
PL136 & 109 & PL167 & 31 & PL83 & 9 & PL205 & 6 & - \\
PL92 & 107 & PL37 & 30 & PL103 & 9 & PL231 & 6 & - \\
PL4 & 104 & PL74 & 30 & PL151 & 9 & PL24 & 5 & - \\
\hline
\end{tabular}

Dataset protect patients' privacy, and is also available online [21]. 